Vahid Dadashi · Mihai Postolache

\title{
Forward-backward splitting algorithm for fixed point problems and zeros of the sum of monotone operators
}

Received: 7 February 2018 / Accepted: 29 December 2018 / Published online: 8 January 2019

(C) The Author(s) 2019

\begin{abstract}
In this paper, we construct a forward-backward splitting algorithm for approximating a zero of the sum of an $\alpha$-inverse strongly monotone operator and a maximal monotone operator. The strong convergence theorem is then proved under mild conditions. Then, we add a nonexpansive mapping in the algorithm and prove that the generated sequence converges strongly to a common element of a fixed points set of a nonexpansive mapping and zero points set of the sum of monotone operators. We apply our main result both to equilibrium problems and convex programming.
\end{abstract}

Mathematics Subject Classification $\quad 47 \mathrm{H} 05 \cdot 47 \mathrm{H} 09$

\section{Introduction}

A very common problem in diverse areas of mathematics and physical sciences consists of finding zero points of some nonlinear operators. For instance, evolution equations, complementarity problems, mini-max problems, variational inequalities and optimization problems; please, see: Kinderlehrer and Stampacchia [19], Kamimura and Takahashi [16], Cho et al. [8], Qin and Su [31] Qin et al. [32] and the references therein. One of the methods of approximating zero points is the proximal point algorithm. This algorithm has been introduced by Martinet [23]. Rockafellar [35] studied the proximal point algorithm for maximal monotone operators to find a zero of the monotone operator. After that, many authors considered this method and studied it and its modified versions in Hilbert and Banach spaces: Burachik and Scheimberg [6], Rouhani and Khatibzadeh [36], Li and Song [20], Alber and Yao [1], Boikanyo and Morosanu [3], Matsushita and Xu [24], Khatibzadeh [18], Dadashi [10,11].

Moreover, Takahashi [39], Yao and Noor [45], Wang and Cui [44], Tian and Wang [41] and Wang and Cui [43] investigated the contraction proximal point algorithm and viscosity approximation method for finding zeros of maximal monotone operators. They proved the strong convergence of this method under some appropriate conditions.

V. Dadashi

Department of Mathematics, Sari Branch, Islamic Azad University, Sari, Iran

E-mail: vahid.dadashi@iausari.ac.ir

M. Postolache

China Medical University, Taichung 40402, Taiwan

M. Postolache

Institute of Mathematical Statistics and Applied Mathematics, Romanian Academy, 050711 Bucharest, Romania

M. Postolache ( $\varangle)$

Department of Mathematics and Computer Science, University Politehnica of Bucharest, 060042 Bucharest, Romania

E-mail: mihai@mathem.pub.ro 
One of the problems of finding zero points of nonlinear operator is to find zero points of the sum of an $\alpha$-inverse strongly monotone operator and a maximal monotone operator. There are various applications of the problem of finding zero points of the sum of two operators; see [12,13,28,33,42] for example and the references therein. Passty [30] introduced an iterative method called forward-backward method for finding zero points of the sum of two operators. Moudafi and Oliny [27] considered an algorithm and proved the generated sequence by their algorithm converges weakly to a zero point of the sum of two maximal monotone operators. See also [25].

In recent years, monotone operators have received a lot of attention for treating zero points of monotone operators and fixed point of mappings which are Lipschitz continuous; see $[7,17,22,26,29,32,40,47,48]$ and the references therein.

Very recently, Boikanyo [4] used proximal point algorithm for finding zero points of the sum of two operators such that the sequence of error terms is square summable in norm.

In this paper, we use and generalize the proximal point algorithm in Yao and Shahzad [46] for finding a point in the intersection of fixed points set of the nonexpansive mapping and zero points set of the sum of monotone operators. For the case when the sequence of error terms converges strongly to zero in norm, we prove that the generated sequence by our algorithm converges strongly to zero in norm.

The paper is organized as follows. Section 2 gathers some definitions and lemmas of geometry of Banach spaces and monotone operators, which will be needed in the remaining sections. In Sect. 3, two iterative algorithms are proposed and strong convergence theorems for finding a point in the intersection of fixed point set of a nonexpansive mapping and zero set of sum of two operators are established. Finally, in Sect. 4, the ideas of Sect. 3 are applied to solve equilibrium problems and find the minimizer of a convex function.

\section{Preliminaries}

In this section, we provide some basic concepts, definitions and lemmas which will be used in the sequel. Let $C$ be a nonempty, closed, and convex subset of a real Hilbert space $H$ with norm $\|\cdot\|$ and inner product $\langle\cdot, \cdot\rangle$. For a mapping $T: C \rightarrow C$, the fixed points set of $T$ is denoted by $F(T)=\{x \in C: T x=x\}$. We write $x_{n} \rightarrow x$ to indicate that the sequence $\left\{x_{n}\right\}$ strongly converges to $x$.

Definition 2.1 A multifunction $B: H \rightrightarrows 2^{H}$ is called a monotone operator if for every $x, y \in H$,

$$
\left\langle x^{*}-y^{*}, x-y\right\rangle \geq 0, \quad \forall x^{*} \in B(x), \quad \forall y^{*} \in B(y) .
$$

A monotone operator $B: H \rightrightarrows 2^{H}$ is said to be maximal monotone, when its graph is not properly included in the graph of any other monotone operator on the same space. The zero points set of $B$ is denoted by $B^{-1}(0)=\{x \in H: 0 \in B x\}$.

Definition 2.2 A single valued operator $A: H \rightarrow H$ is called $\alpha$-inverse strongly monotone for a positive number $\alpha$ if

$$
\langle A x-A y, x-y\rangle \geq \alpha\|A x-A y\|^{2}, \quad \forall x, y \in H .
$$

It is easy to see that every $\alpha$-inverse strongly monotone is monotone and continuous.

Lemma 2.3 [29] Let $C$ be a nonempty, closed, and convex subset of a real Hilbert space H. Let the mapping $A: C \rightarrow H$ be $\alpha$-inverse strongly monotone and $\lambda>0$ be a constant. Then, we have

$$
\|(I-\lambda A) x-(I-\lambda A) y\|^{2} \leq\|x-y\|^{2}+\lambda(\lambda-2 \alpha)\|A x-A y\|^{2}
$$

for all $x, y \in C$. In particular, if $0<\lambda \leq 2 \alpha$, then $I-\lambda A$ is nonexpansive.

Remark 2.4 Let $H$ be a Hilbert space and $B: H \rightrightarrows 2^{H}$ be a maximal monotone operator. By Theorem 3.4 of Chapter 5 of [9], the equation $0 \in \lambda B \tilde{x}+(\tilde{x}-x)$ has a unique solution $x_{\lambda} \in D(B)$ for every $x \in H$. The operator $J_{\lambda}: H \rightarrow D(B)$ defined by $J_{\lambda}(x)=x_{\lambda}$ is called the resolvent of $B$ of order $\lambda$, which $x_{\lambda}$ satisfies $\frac{1}{\lambda}\left(x-x_{\lambda}\right) \in B\left(x_{\lambda}\right)$. Therefore, $\frac{1}{\lambda}\left(x-J_{\lambda}(x)\right) \in B\left(J_{\lambda}(x)\right)$. Since $B$ is maximal monotone, it is easy to see that $J_{\lambda}$ is firmly nonexpansive, and $F\left(J_{\lambda}\right)=(B)^{-1}(0)$. 
Lemma 2.5 [2] Let $C$ be a nonempty, closed, and convex subset of a real Hilbert space $H$ and $A: C \rightarrow H$ an operator. If $B: H \rightarrow 2^{H}$ is a maximal monotone operator, then

$$
F\left(J_{\lambda}(I-\lambda A)\right)=(A+B)^{-1}(0) .
$$

Lemma 2.6 [5] For $\lambda>0, \mu>0$ and $x \in H$,

$$
J_{\lambda} x=J_{\mu}\left(\frac{\mu}{\lambda} x+\left(1-\frac{\mu}{\lambda}\right) J_{\lambda} x\right) .
$$

Let $C$ be a convex closed subset of $H$. The operator $P_{C}$ is called a metric projection operator if it assigns to each $x \in H$ its nearest point $y \in C$ such that

$$
\|x-y\|=\min \{\|x-z\|: z \in C\} .
$$

The element $y$ above is called the metric projection of $H$ onto $C$ and denoted by $P_{C} x$. It exists and is unique at any point of the reflexive strictly convex space.

Lemma 2.7 Let $H$ be a Hilbert space and $C$ is a nonempty, closed and convex subset of $H$. Then, for all $x \in H$, the element $z=P_{C} x$ if and only if

$$
\langle x-z, z-y\rangle \geq 0, \quad \forall y \in C .
$$

Lemma 2.8 [38] Let $\left\{x_{n}\right\}$ and $\left\{y_{n}\right\}$ be bounded sequences in a Banach space $X$ and $\left\{\beta_{n}\right\}$ be a sequence in $[0,1]$ with $0<\liminf _{n \rightarrow \infty} \beta_{n} \leq \limsup _{n \rightarrow \infty} \beta_{n}<1$. Suppose that $x_{n+1}=\left(1-\beta_{n}\right) y_{n}+\beta_{n} x_{n}$ for all $n \geq 0$ and $\lim \sup _{n \rightarrow \infty}\left(\left\|y_{n+1}-y_{n}\right\|-\left\|x_{n+1}-x_{n}\right\|\right) \leq 0$. Then, $\lim _{n \rightarrow \infty}\left\|x_{n}-y_{n}\right\| \leq 0$.

Lemma 2.9 [14] Let $C$ be a nonempty, closed, and convex subset of $H$. Let $S: C \rightarrow C$ be a nonexpansive mapping. Then, the mapping $I-S$ is demiclosed at zero, that is, if $\left\{x_{n}\right\}$ is a sequence in $C$ such that $x_{n} \rightarrow x$ and $\left\|x_{n}-S x_{n}\right\| \rightarrow 0$, then $x \in F(S)$.

Lemma 2.10 [37] Suppose that $H$ is a real Hilbert space and $0<p \leq t_{n} \leq q<1$ for all $n \geq 1$. Suppose further that $\left\{x_{n}\right\}$ and $\left\{y_{n}\right\}$ are sequences of $H$ such that

$$
\limsup _{n \rightarrow \infty}\left\|x_{n}\right\| \leq r, \quad \limsup _{n \rightarrow \infty}\left\|y_{n}\right\| \leq r, \quad \text { and } \quad \lim _{n \rightarrow \infty}\left\|t_{n} x_{n}+\left(1-t_{n}\right) y_{n}\right\|=r
$$

hold for some $r \geq 0$. Then, $\lim _{n \rightarrow \infty}\left\|x_{n}-y_{n}\right\|=0$.

Lemma 2.11 [21] Assume that $\left\{s_{n}\right\}$ is a sequence of nonnegative numbers such that

$$
s_{n+1} \leq\left(1-\gamma_{n}\right) s_{n}+\delta_{n}, \quad \forall n \geq 0,
$$

where $\left\{\gamma_{n}\right\}$ is a sequence in $(0,1)$ and $\left\{\delta_{n}\right\}$ is a sequence in $\mathbb{R}$ such that

(i) $\sum_{n=1}^{\infty} \gamma_{n}=\infty$,

(ii) $\limsup _{n \rightarrow \infty} \frac{\delta_{n}}{\gamma_{n}} \leq 0$ or $\sum_{n=1}^{\infty}\left|\delta_{n}\right|<\infty$.

Then $\lim _{n \rightarrow \infty} s_{n}=0$.

\section{Main results}

In this section, using the forward-backward splitting algorithm we prove some strong convergence theorems for approximating a zero of the sum of an $\alpha$-inverse strongly monotone operator and a maximal monotone operator.

To prove the first result, we use the technique developed by Yao and Shahzad [46].

Theorem 3.1 Let $C$ be a nonempty, closed and convex subset of a real Hilbert space $H$. Let $A: C \rightarrow H$ be an $\alpha$-inverse strongly monotone mapping and $B$ be maximal monotone operator of $H$ into $2^{H}$ such that the domain of $B$ is included in $C$ and $(A+B)^{-1}(0) \neq \emptyset$. Assume that $J_{\lambda}$ is the resolvent of $B$ for $\lambda>0$. Let the sequence $\left\{z_{n}\right\}$ generated as:

$$
\left\{\begin{array}{l}
w_{n}=J_{\lambda_{n}}\left(z_{n}-\lambda_{n} A z_{n}\right) \\
z_{n+1}=\alpha_{n} z_{n}+\beta_{n} w_{n}+\gamma_{n} e_{n}
\end{array}\right.
$$

where $e_{n}$ is an error vector, $z_{1} \in H, \alpha_{n}, \beta_{n}, \gamma_{n} \in(0,1)$ and $\alpha_{n}+\beta_{n}+\gamma_{n}=1$. Suppose the control sequences satisfy the following conditions: 
(a) $\lim _{n \rightarrow \infty} \gamma_{n}=0$, and $\sum_{n=1}^{\infty} \gamma_{n}=\infty$;

(b) $e_{n} \rightarrow 0$;

(c) $0<a \leq \alpha_{n} \leq b<1$ and $0<c \leq \beta_{n} \leq d<1$;

(d) $0<\varepsilon \leq \lambda_{n}<2 \alpha$ and $\lim _{n \rightarrow \infty}\left(\bar{\lambda}_{n}-\lambda_{n+1}\right)=0$.

Then, $\left\{z_{n}\right\}$ converges strongly to the point $q \in(A+B)^{-1}(0)$, where $q=P_{(A+B)^{-1}(0)}(0)$.

Proof First of all, we shall show that $\left\{z_{n}\right\}$ generated by algorithm (3.1) is bounded. Fix $p \in(A+B)^{-1}(0)$, by the fact that $J_{\lambda_{n}}$ is nonexpansive and Lemma 2.3, we obtain

$$
\begin{aligned}
\left\|w_{n}-p\right\| & =\left\|J_{\lambda_{n}}\left(z_{n}-\lambda_{n} A z_{n}\right)-J_{\lambda_{n}}\left(p-\lambda_{n} A p\right)\right\| \\
& \leq\left\|\left(I-\lambda_{n} A\right) z_{n}-\left(I-\lambda_{n} A\right) p\right\| \\
& \leq\left\|z_{n}-p\right\| .
\end{aligned}
$$

It follows that

$$
\begin{aligned}
\left\|z_{n+1}-p\right\| & \leq \alpha_{n}\left\|z_{n}-p\right\|+\beta_{n}\left\|w_{n}-p\right\|+\gamma_{n}\left\|e_{n}-p\right\| \\
& \leq\left(1-\gamma_{n}\right)\left\|z_{n}-p\right\|+\gamma_{n}\left(\left\|e_{n}\right\|+\|p\|\right) \\
& \leq\left(1-\gamma_{n}\right)\left\|z_{n}-p\right\|+\gamma_{n} M_{1} \\
& \leq \max \left\{\left\|z_{n}-p\right\|, M_{1}\right\},
\end{aligned}
$$

where $\sup \left\|e_{n}\right\|+\|p\| \leq M_{1}$ for some $M_{1}>0$. By induction on $n$, we obtain that

$$
\left\|z_{n+1}-p\right\| \leq \max \left\{\left\|z_{1}-p\right\|, M_{1}\right\}
$$

Hence, the sequence $\left\{z_{n}\right\}$ is bounded and so is $\left\{w_{n}\right\}$.

Define $z_{n+1}=\alpha_{n} z_{n}+\left(1-\alpha_{n}\right) v_{n}$ for all $n \geq 0$. Then, we obtain from (3.1),

$$
\begin{aligned}
v_{n+1}-v_{n}= & \frac{z_{n+2}-\alpha_{n+1} z_{n+1}}{1-\alpha_{n+1}}-\frac{z_{n+1}-\alpha_{n} z_{n}}{1-\alpha_{n}} \\
= & \frac{\beta_{n+1} w_{n+1}+\gamma_{n+1} e_{n+1}}{1-\alpha_{n+1}}-\frac{\beta_{n} w_{n}+\gamma_{n} e_{n}}{1-\alpha_{n}} \\
= & \frac{\beta_{n+1}}{1-\alpha_{n+1}}\left(w_{n+1}-w_{n}\right)+\left(\frac{\beta_{n+1}}{1-\alpha_{n+1}}-\frac{\beta_{n}}{1-\alpha_{n}}\right) w_{n} \\
& +\frac{\gamma_{n+1} e_{n+1}}{1-\alpha_{n+1}}-\frac{\gamma_{n} e_{n}}{1-\alpha_{n}} .
\end{aligned}
$$

Set $u_{n}=\left(I-\lambda_{n} A\right) z_{n}$. It follows from Lemma 2.3 that

$$
\left\|u_{n+1}-u_{n}\right\| \leq\left\|z_{n+1}-z_{n}\right\| .
$$

If $\lambda_{n} \leq \lambda_{n+1}$, by Lemma 2.6, we have

$$
w_{n+1}=J_{\lambda_{n+1}}\left(u_{n+1}\right)=J_{\lambda_{n}}\left(\frac{\lambda_{n}}{\lambda_{n+1}} u_{n+1}+\left(1-\frac{\lambda_{n}}{\lambda_{n+1}}\right) J_{\lambda_{n+1}}\left(u_{n+1}\right)\right),
$$

and hence, we get,

$$
\begin{aligned}
\left\|w_{n+1}-w_{n}\right\| & =\left\|J_{\lambda_{n+1}}\left(u_{n+1}\right)-J_{\lambda_{n}}\left(u_{n}\right)\right\| \\
& \leq \frac{\lambda_{n}}{\lambda_{n+1}}\left\|u_{n+1}-u_{n}\right\|+\left(1-\frac{\lambda_{n}}{\lambda_{n+1}}\right)\left\|w_{n+1}-u_{n}\right\| \\
& \leq\left\|z_{n+1}-z_{n}\right\|+\frac{1}{\varepsilon}\left|\lambda_{n+1}-\lambda_{n}\right|\left\|w_{n+1}-u_{n}\right\| .
\end{aligned}
$$


If $\lambda_{n}>\lambda_{n+1}$, again using Lemma 2.6, we obtain

$$
\begin{aligned}
\left\|w_{n}-w_{n+1}\right\| & =\left\|J_{\lambda_{n}}\left(u_{n}\right)-J_{\lambda_{n+1}}\left(u_{n+1}\right)\right\| \\
& \leq \frac{\lambda_{n+1}}{\lambda_{n}}\left\|u_{n}-u_{n+1}\right\|+\left(1-\frac{\lambda_{n+1}}{\lambda_{n}}\right)\left\|w_{n}-u_{n+1}\right\| \\
& \leq\left\|z_{n+1}-z_{n}\right\|+\frac{1}{\varepsilon}\left|\lambda_{n+1}-\lambda_{n}\right|\left\|w_{n}-u_{n+1}\right\| .
\end{aligned}
$$

Therefore, from (3.2) and (3.3), we imply that

$$
\left\|w_{n+1}-w_{n}\right\| \leq\left\|z_{n+1}-z_{n}\right\|+\frac{M_{2}}{\varepsilon}\left|\lambda_{n+1}-\lambda_{n}\right|
$$

where, $M_{2}$ satisfies

$$
\sup \left\{\left\|w_{n+1}-u_{n}\right\|,\left\|w_{n}\right\|+\left\|u_{n+1}\right\|, \quad n \geq 0\right\} \leq M_{2} .
$$

Hence, we get

$$
\begin{aligned}
\left\|v_{n+1}-v_{n}\right\| \leq & \frac{\beta_{n+1}}{1-\alpha_{n+1}}\left\|w_{n+1}-w_{n}\right\|+\left|\frac{\beta_{n+1}}{1-\alpha_{n+1}}-\frac{\beta_{n}}{1-\alpha_{n}}\right|\left\|w_{n}\right\| \\
& +\frac{\gamma_{n+1}\left\|e_{n+1}\right\|}{1-\alpha_{n+1}}+\frac{\gamma_{n}\left\|e_{n}\right\|}{1-\alpha_{n}} \\
\leq & \frac{\beta_{n+1}}{1-\alpha_{n+1}}\left\|z_{n+1}-z_{n}\right\|+\frac{\beta_{n+1}}{1-\alpha_{n+1}} \frac{M_{2}}{\varepsilon}\left|\lambda_{n+1}-\lambda_{n}\right| \\
& +\left|\frac{\beta_{n+1}}{1-\alpha_{n+1}}-\frac{\beta_{n}}{1-\alpha_{n}}\right| M_{2}+\left(\frac{\gamma_{n+1}}{1-\alpha_{n+1}}+\frac{\gamma_{n}}{1-\alpha_{n}}\right) M_{1},
\end{aligned}
$$

which implies that

$$
\begin{aligned}
& \lim \sup \left(\left\|v_{n+1}-v_{n}\right\|-\left\|z_{n+1}-z_{n}\right\|\right) \\
& \quad \leq \limsup \left[\frac{\beta_{n+1}}{1-\alpha_{n+1}} \frac{M_{2}}{\varepsilon}\left|\lambda_{n+1}-\lambda_{n}\right|+\left|\frac{\beta_{n+1}}{1-\alpha_{n+1}}-\frac{\beta_{n}}{1-\alpha_{n}}\right| M_{2}+\frac{\gamma_{n+1}}{1-\alpha_{n+1}} M_{1}+\frac{\gamma_{n}}{1-\alpha_{n}} M_{1}\right], \\
& \quad \leq \lim \sup \left[\frac{\beta_{n+1}}{1-\alpha_{n+1}} \frac{M_{2}}{\varepsilon}\left|\lambda_{n+1}-\lambda_{n}\right|+\left|\frac{\gamma_{n+1}}{1-\alpha_{n+1}}-\frac{\gamma_{n}}{1-\alpha_{n}}\right| M_{2}+\frac{\gamma_{n+1}}{1-\alpha_{n+1}} M_{1}+\frac{\gamma_{n}}{1-\alpha_{n}} M_{1}\right] \\
& =0 .
\end{aligned}
$$

By Lemma 2.8, we have

$$
\lim _{n \rightarrow \infty}\left\|z_{n}-v_{n}\right\|=0
$$

and, hence,

$$
\lim _{n \rightarrow \infty}\left\|z_{n+1}-z_{n}\right\|=\lim _{n \rightarrow \infty}\left(1-\gamma_{n}\right)\left\|v_{n}-z_{n}\right\|=0 .
$$

Also, we have

$$
\begin{aligned}
\left\|z_{n}-w_{n}\right\| & \leq\left\|z_{n}-z_{n+1}\right\|+\left\|z_{n+1}-w_{n}\right\| \\
& \leq\left\|z_{n}-z_{n+1}\right\|+\alpha_{n}\left\|z_{n}-w_{n}\right\|+\gamma_{n}\left\|e_{n}-w_{n}\right\| .
\end{aligned}
$$

Therefore

$$
\left\|z_{n}-w_{n}\right\| \leq \frac{1}{1-\alpha_{n}}\left\|z_{n}-z_{n+1}\right\|+\frac{\gamma_{n}}{1-\alpha_{n}}\left\|e_{n}-w_{n}\right\|,
$$

which implies that

$$
\lim _{n \rightarrow \infty}\left\|z_{n}-w_{n}\right\|=0
$$


Next, we show that $w_{w}\left(z_{n}\right) \subset(A+B)^{-1}(0)$.

Let $p \in w_{w}\left(z_{n}\right)$ Then, there exists a subsequence $\left\{z_{n_{j}}\right\}$ converging weakly to $p$. Since $J_{\lambda_{n_{j}}}$ is resolvent of $B$, by definition of $w_{n}$, we have that

$$
\frac{z_{n_{j}}-w_{n_{j}}}{\lambda_{n_{j}}}-A z_{n_{j}} \in B w_{n_{j}}
$$

By monotonicity of $B$, we obtain

$$
0 \leq\left\langle\frac{z_{n_{j}}-w_{n_{j}}}{\lambda_{n_{j}}}-A z_{n_{j}}-w, w_{n_{j}}-u\right\rangle
$$

for each $(u, w) \in B$. Taking limit in (3.4) and $j \rightarrow \infty$, it follows that $\langle 0-A p-w, p-u\rangle \geq 0$. Maximal monotonicity of $B$ implies that $-A p \in B p$ and hence $p \in(A+B)^{-1}(0)$.

Set $q=P_{(A+B)^{-1}(0)}(0)$ and take a subsequence $\left\{z_{n_{j}}\right\}$ of $\left\{z_{n}\right\}$ such that $z_{n_{j}} \rightarrow z$ and

$$
\limsup _{n \rightarrow \infty}\left\langle q, q-z_{n}\right\rangle=\lim _{j \rightarrow \infty}\left\langle q, q-z_{n_{j}}\right\rangle .
$$

Thus, $z \in(A+B)^{-1}(0)$ and hence

$$
\limsup _{n \rightarrow \infty}\left\langle q, q-z_{n}\right\rangle=\langle q, q-z\rangle \leq 0
$$

Finally, we show that the sequence $\left\{z_{n}\right\}$ converges strongly to $q=P_{(A+B)^{-1}(0)}(0)$.

By (3.1), we have

$$
\begin{aligned}
\left\|z_{n+1}-q\right\|^{2} \leq & \left\|\alpha_{n}\left(z_{n}-q\right)+\beta_{n}\left(w_{n}-q\right)-\gamma_{n} q+\gamma_{n} e_{n}\right\|^{2} \\
\leq & {\left[\left\|\alpha_{n}\left(z_{n}-q\right)+\beta_{n}\left(w_{n}-q\right)-\gamma_{n} q\right\|+\gamma_{n}\left\|e_{n}\right\|\right]^{2} } \\
= & \left\|\alpha_{n}\left(z_{n}-q\right)+\beta_{n}\left(w_{n}-q\right)-\gamma_{n} q\right\|^{2} \\
& +\gamma_{n}\left\|e_{n}\right\|\left[\gamma_{n}\left\|e_{n}\right\|+2\left\|\alpha_{n}\left(z_{n}-q\right)+\beta_{n}\left(w_{n}-q\right)-\gamma_{n} q\right\|\right] \\
\leq & \left\|\alpha_{n}\left(z_{n}-q\right)+\beta_{n}\left(w_{n}-q\right)\right\|^{2}-2 \gamma_{n}\left\langle q, z_{n+1}-q-\gamma_{n} e_{n}\right\rangle+M_{3} \gamma_{n}\left\|e_{n}\right\| \\
\leq & \left(\alpha_{n}\left\|z_{n}-q\right\|+\beta_{n}\left\|w_{n}-q\right\|\right)^{2}-2 \gamma_{n}\left\langle q, z_{n+1}-q\right\rangle+2 \gamma_{n}^{2}\left\|e_{n}\right\|\|q\|+M_{3} \gamma_{n}\left\|e_{n}\right\| \\
\leq & \left(1-\gamma_{n}\right)\left\|z_{n}-q\right\|^{2}+\gamma_{n}\left[2\left\langle q, q-z_{n+1}\right\rangle+\left(2\|q\|+M_{3}\right) \gamma_{n}\left\|e_{n}\right\|\right] \\
= & \left(1-\gamma_{n}\right)\left\|z_{n}-q\right\|^{2}+\delta_{n},
\end{aligned}
$$

where $M_{3}$ satisfies in

$$
\sup \left\{\left\|e_{n}\right\|+2\left\|\alpha_{n}\left(z_{n}-q\right)+\beta_{n}\left(w_{n}-q\right)-\gamma_{n} q\right\|, \quad n \geq 0\right\} \leq M_{3} .
$$

Then, Lemma 2.11 implies that $z_{n} \rightarrow q$ as $n \rightarrow \infty$, and this completes the proof.

If we take $A=0$ in Theorem 3.1, then we obtain the following result.

Corollary 3.2 Let $C$ be a nonempty closed convex subset of a real Hilbert space H. Let $B$ be maximal monotone operator of $H$ into $2^{H}$ such that the domain of $B$ is included in $C$ and $B^{-1}(0) \neq \emptyset$. Assume that $J_{\lambda}$ is the resolvent of $B$ for $\lambda>0$. Let the sequence $\left\{z_{n}\right\}$ generated by the following algorithm:

$$
z_{n+1}=\alpha_{n} z_{n}+\beta_{n} J_{\lambda_{n}} z_{n}+\gamma_{n} e_{n},
$$

where $z_{1} \in H$ and $\alpha_{n}, \beta_{n}, \gamma_{n} \in(0,1)$ and $\alpha_{n}+\beta_{n}+\gamma_{n}=1$. Suppose that the control sequences satisfy in the conditions (a), (b), (c) and (d') $0<\varepsilon \leq \lambda_{n}$ and $\lim _{n \rightarrow \infty}\left(\lambda_{n}-\lambda_{n+1}\right)=0$.

Then, $\left\{z_{n}\right\}$ converges strongly to a point $q \in B^{-1}(0)$, where $q=P_{B^{-1}(0)}(0)$. 
Theorem 3.3 Let $C$ be a nonempty closed convex subset of a real Hilbert space H. Suppose that $S: C \rightarrow C$ is a nonexpansive mapping, $A: C \rightarrow H$ an $\alpha$-inverse strongly monotone mapping and $B$ a maximal monotone operator of $H$ into $2^{H}$ such that the domain of $B$ is included in $C$ and $F(S) \cap(A+B)^{-1}(0) \neq \emptyset$. Assume that $J_{\lambda}$ is the resolvent of $B$ for $\lambda>0$. Let the sequence $\left\{x_{n}\right\}$ generated by the following algorithm:

$$
\left\{\begin{array}{l}
y_{n}=J_{\lambda_{n}}\left(x_{n}-\lambda_{n} A x_{n}\right), \\
x_{n+1}=\alpha_{n} S x_{n}+\beta_{n} y_{n}+\gamma_{n} e_{n}
\end{array}\right.
$$

where $x_{1} \in H$ and $\alpha_{n}, \beta_{n}, \gamma_{n} \in(0,1)$ and $\alpha_{n}+\beta_{n}+\gamma_{n}=1$. Suppose that the control sequences satisfy in the conditions (a), (b), (c), (d) and (e) either $\sum \alpha_{n}<\infty$ or $\frac{\alpha_{n}}{\gamma_{n}} \rightarrow 0$.

Then, $\left\{x_{n}\right\}$ converges strongly to a point $q \in F(S) \cap(A+B)^{-1}(0)$, where $q=P_{F(S) \cap(A+B)^{-1}(0)}(0)$.

Proof At first, we show that $\left\{x_{n}\right\}$ generated by algorithm (3.5) is bounded. Similar to (3.2), we have

$$
\left\|y_{n}-p\right\| \leq\left\|x_{n}-p\right\|,
$$

for each $p \in(A+B)^{-1}(0)$. Fix $p \in F(S) \cap(A+B)^{-1}(0)$. By (3.5), we get

$$
\begin{aligned}
\left\|x_{n+1}-p\right\| & \leq \alpha_{n}\left\|S x_{n}-p\right\|+\beta_{n}\left\|y_{n}-p\right\|+\gamma_{n}\left\|e_{n}-p\right\| \\
& \leq\left(1-\gamma_{n}\right)\left\|x_{n}-p\right\|+\gamma_{n}\left(\left\|e_{n}\right\|+\|p\|\right) \\
& \leq\left(1-\gamma_{n}\right)\left\|x_{n}-p\right\|+\gamma_{n} M \\
& \leq \max \left\{\left\|x_{n}-p\right\|, M\right\},
\end{aligned}
$$

where sup $\left\|e_{n}\right\|+\|p\| \leq M$ for some $M>0$. By induction on $n$, we obtain that

$$
\left\|x_{n+1}-p\right\| \leq \max \left\{\left\|x_{1}-p\right\|, M\right\} .
$$

Hence, the sequence $\left\{x_{n}\right\}$ is bounded and so are $\left\{y_{n}\right\}$ and $\left\{S x_{n}\right\}$.

Let the sequence $\left\{z_{n}\right\}$ generated by (3.1). We prove that $\left\{x_{n}\right\}$ and $\left\{z_{n}\right\}$ are equivalent. Since $J_{\lambda_{n}}$ is nonexpansive and by Lemma 2.3, we obtain

$$
\begin{aligned}
\left\|y_{n}-w_{n}\right\| & =\left\|J_{\lambda_{n}}\left(x_{n}-\lambda_{n} A x_{n}\right)-J_{\lambda_{n}}\left(z_{n}-\lambda_{n} A z_{n}\right)\right\| \\
& \leq\left\|\left(I-\lambda_{n} A\right) x_{n}-\left(I-\lambda_{n} A\right) z_{n}\right\| \\
& \leq\left\|x_{n}-z_{n}\right\|,
\end{aligned}
$$

and hence,

$$
\begin{aligned}
\left\|x_{n+1}-z_{n+1}\right\| & \leq \alpha_{n}\left\|S x_{n}-z_{n}\right\|+\beta_{n}\left\|y_{n}-w_{n}\right\| \\
& \leq \alpha_{n}\left\|S x_{n}-y_{n}\right\|+\alpha_{n}\left\|y_{n}-w_{n}\right\|+\alpha_{n}\left\|w_{n}-z_{n}\right\|+\beta_{n}\left\|y_{n}-w_{n}\right\| \\
& \leq\left(\alpha_{n}+\beta_{n}\right)\left\|x_{n}-z_{n}\right\|+\alpha_{n}\left(\left\|S x_{n}-y_{n}\right\|+\left\|w_{n}-z_{n}\right\|\right) \\
& \leq\left(1-\gamma_{n}\right)\left\|x_{n}-z_{n}\right\|+\alpha_{n}\left(\left\|S x_{n}-y_{n}\right\|+\left\|w_{n}-z_{n}\right\|\right) .
\end{aligned}
$$

Applying Lemma 2.11 with conditions (a) and (e), we conclude that $\left\|x_{n}-z_{n}\right\| \rightarrow 0$. Using Theorem 3.1, we imply that $x_{n} \rightarrow q=P_{(A+B)^{-1}(0)}(0)$ and so $y_{n} \rightarrow q$ by (3.6).

To finish our proof, it suffices to show that $q=P_{F(S) \cap(A+B)^{-1}(0)}(0)$. We notice that

$$
\begin{aligned}
\left\|S x_{n}-p+\gamma_{n}\left(e_{n}-S x_{n}\right)\right\| & \leq\left\|S x_{n}-p\right\|+\gamma_{n}\left\|e_{n}-S x_{n}\right\| \\
& \leq\left\|x_{n}-p\right\|+\gamma_{n}\left\|e_{n}-S x_{n}\right\| .
\end{aligned}
$$

This implies from the conditions that

$$
\limsup _{n \rightarrow \infty}\left\|S x_{n}-p+\gamma_{n}\left(e_{n}-S x_{n}\right)\right\| \leq\|q-p\| .
$$

We also have

$$
\begin{aligned}
\left\|y_{n}-p+\gamma_{n}\left(e_{n}-S x_{n}\right)\right\| & \leq\left\|y_{n}-p\right\|+\gamma_{n}\left\|e_{n}-S x_{n}\right\| \\
& \leq\left\|x_{n}-p\right\|+\gamma_{n}\left\|e_{n}-S x_{n}\right\| .
\end{aligned}
$$


Therefore

$$
\limsup _{n \rightarrow \infty}\left\|y_{n}-p+\gamma_{n}\left(e_{n}-S x_{n}\right)\right\| \leq\|q-p\| \text {. }
$$

On the other hand, we have

$$
\begin{aligned}
\lim _{n \rightarrow \infty}\left\|\beta_{n}\left(y_{n}-p+\gamma_{n}\left(e_{n}-S x_{n}\right)\right)+\left(1-\beta_{n}\right)\left(S x_{n}-p+\gamma_{n}\left(e_{n}-S x_{n}\right)\right)\right\| & =\lim _{n \rightarrow \infty}\left\|x_{n+1}-p\right\| \\
& =\|q-p\| .
\end{aligned}
$$

It follows from Lemma 2.10 that $\left\|S x_{n}-y_{n}\right\| \rightarrow 0$. Then, we obtain

$$
\left\|S x_{n}-x_{n}\right\| \leq\left\|S x_{n}-y_{n}\right\|+\left\|y_{n}-x_{n}\right\| \rightarrow 0 .
$$

From Lemma 2.9, we conclude that $q \in F(S)$. This together with $q=P_{(A+B)^{-1}(0)}(0)$ implies that $q=$ $P_{F(S) \cap(A+B)^{-1}(0)}(0)$ and hence $\left\{x_{n}\right\}$ converges strongly to $q=P_{F(S) \cap(A+B)^{-1}(0)}(0)$.

If we take $A=0$ in Theorem 3.3, then we obtain the following result.

Corollary 3.4 Let $C$ be a nonempty closed convex subset of a real Hilbert space $H$. Let $S: C \rightarrow C$ be a nonexpansive mapping and $B$ be a maximal monotone operator of $H$ into $2^{H}$ such that the domain of $B$ is included in $C$ and $F(S) \cap B^{-1}(0) \neq \emptyset$. Assume that $J_{\lambda}$ be the resolvent of $B$ for $\lambda>0$. Let the sequence $\left\{x_{n}\right\}$ generated by the following algorithm:

$$
x_{n+1}=\alpha_{n} S x_{n}+\beta_{n} J_{\lambda_{n}} x_{n}+\gamma_{n} e_{n}
$$

where $x_{1} \in H$ and $\alpha_{n}, \beta_{n}, \gamma_{n} \in(0,1)$ and $\alpha_{n}+\beta_{n}+\gamma_{n}=1$. If the control sequences satisfy in the conditions (a), (b), (c), ( $\left.\mathrm{d}^{\prime}\right)$ and (e), then $\left\{x_{n}\right\}$ converges strongly to a point $q \in F(S) \cap B^{-1}(0)$, where $q=P_{F(S) \cap B^{-1}(0)}(0)$.

Corollary 3.5 Let $H$ be a real Hilbert space. Suppose that $B$ and $M$ be maximal monotone operators of $H$ into $2^{H}$ such that $M^{-1}(0) \cap B^{-1}(0) \neq \emptyset$. Assume that $J_{\lambda}^{B}$ and $J_{r}^{M}$ be the resolvent of $B$ and $M$ for $\lambda>0$ and $r>0$, respectively. Let the sequence $\left\{x_{n}\right\}$ generated by the following algorithm:

$$
x_{n+1}=\alpha_{n} J_{r}^{M} x_{n}+\beta_{n} J_{\lambda_{n}}^{B} x_{n}+\gamma_{n} e_{n},
$$

where $x_{1} \in H$ and $\alpha_{n}, \beta_{n}, \gamma_{n} \in(0,1)$ and $\alpha_{n}+\beta_{n}+\gamma_{n}=1$. Suppose that the control sequences satisfy in the conditions (a), (b), (c), (d) and (e) either $\sum \alpha_{n}<\infty$ or $\frac{\alpha_{n}}{\gamma_{n}} \rightarrow 0$.

Then, $\left\{x_{n}\right\}$ converges strongly to a point $q \in M^{-1}(0) \cap B^{-1}(0)$, where $q=P_{M^{-1}(0) \cap B^{-1}(0)}(0)$.

\section{Applications}

Our aim in this section is to discuss an application of our results both to equilibrium problems and convex programming. In this respect, suppose $C$ be a nonempty subset of $H$. A function $F: C \times C \rightarrow \mathbb{R}$ is called bifunction if $F(x, x)=0$ for all $x \in C$. We consider the problem of finding a solution $z \in X$ of

$$
F(z, y) \geq 0, \quad \forall y \in C .
$$

The bifunction $F: C \times C \rightarrow \mathbb{R}$ is called monotone if $F(x, y)+F(y, x) \leq 0$, for all $x, y \in C$. Hadjisavvas and Khatibzadeh [15] introduced a monotone operator $B^{F}$ to each monotone bifunction $F$ by

$$
B^{F}(x)= \begin{cases}\left\{x^{*} \in X^{*}: F(x, y) \geq\left\langle x^{*}, y-x\right\rangle, \forall y \in C\right\}, & x \in C \\ \emptyset, & x \in X \backslash C .\end{cases}
$$

The monotone bifunction $F$ is said to be maximal monotone if $B^{F}$ is maximal monotone. It is obvious that $\bar{x} \in C$ is a solution of an equilibrium problem (4.1) for $F$ if and only if $0 \in B^{F}(\bar{x})$.

The following theorems have been proved in [15] for the maximality of bifunction $F$.

Theorem 4.1 Let $C \subseteq X$ be nonempty, closed and convex. If $F$ is monotone, $F(\cdot, y)$ is upper hemicontinuous (i.e., upper semicontinuous on line segments) for all $y \in C$ and $F(x, \cdot)$ is convex and lower semicontinuous for all $x \in C$, then $F$ is maximal monotone. 
Theorem 4.2 A monotone bifunction $F$ is maximal monotone if and only if for each $\lambda>0$ (equivalently, for some $\lambda>0$ ) and each $x \in H$ there exists $x_{\lambda} \in C$ such that

$$
\lambda F\left(x_{\lambda}, y\right)+\left\langle y-x_{\lambda}, x_{\lambda}-x\right\rangle \geq 0, \quad \forall y \in C .
$$

This element $x_{\lambda}$ is uniquely defined.

Note that (4.2) implies that for every $x \in X, J_{\lambda}^{B^{F}}(x)=x_{\lambda}$ and $x_{\lambda} \in D\left(B^{F}\right)$. Therefore, if $F$ satisfies the assumptions of Theorem 4.1. Then, by (4.2) and for each $n \in \mathbb{N}$ there exist $y_{n}, x_{n} \in X$ which satisfy

$$
\left\{\begin{array}{l}
\lambda_{n} F\left(y_{n}, y\right)+\left\langle y-y_{n}, y_{n}-x_{n}\right\rangle \geq 0, \quad \forall y \in C \\
x_{n+1}=\alpha_{n} S x_{n}+\beta_{n} y_{n}+\gamma_{n} e_{n}
\end{array}\right.
$$

which is equivalent to (3.5) with $B^{F}$ instead of $B$ and $A=0$. Therefore, every convergence result for the sequence generated by (3.5) is true for the sequence generated by (4.3). Then, we get $p$ is a common solution of fixed point set $F(S)$ and equilibrium problem (4.1), whenever $\left\{x_{n}\right\}$ converges strongly to $p \in$ $F(S) \cap\left(B^{F}\right)^{-1}(0)$. In fact, we have the following theorem:

Theorem 4.3 Let $C$ be a nonempty, closed and convex subset of a real Hilbert space $H$. Suppose that $S: C \rightarrow$ $C$ is a nonexpansive mapping, and $F: C \times C \rightarrow \mathbb{R}$ is a monotone bifunction such that $F(\cdot, y)$ is upper hemicontinuous for all $y \in C$ and $F(x, \cdot)$ is convex and lower semicontinuous for all $x \in C$ such that $F(S) \cap E P(F) \neq \emptyset$. Let the sequence $\left\{x_{n}\right\}$ generated by the following algorithm:

$$
\left\{\begin{array}{l}
\lambda_{n} F\left(y_{n}, y\right)+\left\langle y-y_{n}, y_{n}-x_{n}\right\rangle \geq 0, \quad \forall y \in C, \\
x_{n+1}=\alpha_{n} S x_{n}+\beta_{n} y_{n}+\gamma_{n} e_{n}
\end{array}\right.
$$

where $x_{1} \in H$ and $\alpha_{n}, \beta_{n}, \gamma_{n} \in(0,1)$ and $\alpha_{n}+\beta_{n}+\gamma_{n}=1$. If the control sequences satisfy the conditions (a), (b), (c), (d) and (e), then $\left\{x_{n}\right\}$ converges strongly to the common solution of fixed point set and equilibrium problem.

Suppose that $f$ is a proper, convex and lower semicontinuous function. The subdifferential $\partial f: H \rightrightarrows 2^{H}$ of $f$ is defined as follows:

$$
\partial f(x)=\{z \in H:\langle y-x, z\rangle \leq f(y)-f(x), \forall y \in H\} .
$$

Then, $\partial f$ is maximal monotone (see [34]). It is obvious that $0 \in \partial f(x)$ if and only if $f(x)=\min _{y \in H} f(y)$.

Theorem 4.4 Let $H$ be a Hilbert space and $f: H \rightarrow(-\infty,+\infty]$ a proper, convex and lower semicontinuous function such that $(\partial f)^{-1}(0) \neq \emptyset$. Assume that the sequence $\left\{z_{n}\right\}$ generated by the following algorithm:

$$
\left\{\begin{array}{l}
w_{n}=\operatorname{argmin}_{z \in X}\left\{f(z)+\frac{1}{2 \lambda_{n}}\left\|z-z_{n}\right\|^{2}\right\}, \\
z_{n+1}=\alpha_{n} z_{n}+\beta_{n} w_{n}+\gamma_{n} e_{n},
\end{array}\right.
$$

where $z_{1} \in H$ and $\alpha_{n}, \beta_{n}, \gamma_{n} \in(0,1)$ and $\alpha_{n}+\beta_{n}+\gamma_{n}=1$. If the control sequences satisfy the conditions (a), (b), (c) and $\left(\mathrm{d}^{\prime}\right)$, then $\left\{z_{n}\right\}$ converges strongly to the minimizer of $f$.

Proof If we take $B=\partial f$ in Corollary 3.2, then we obtain the desired conclusion immediately.

Open Access This article is distributed under the terms of the Creative Commons Attribution 4.0 International License (http:// creativecommons.org/licenses/by/4.0/), which permits unrestricted use, distribution, and reproduction in any medium, provided you give appropriate credit to the original author(s) and the source, provide a link to the Creative Commons license, and indicate if changes were made. 


\section{References}

1. Alber, Y.; Yao, J.C.: Another version of the proximal point algorithm in a Banach space. Nonlinear Anal. 70, 3159-3171 (2009)

2. Aoyama, K.; Kimura, Y.; Takahashi, W.; Toyoda, M.: On a strongly nonexpansive sequence in Hilbert spaces. J. Nonlinear Convex Anal. 8, 471-489 (2007)

3. Boikanyo, O.A.; Morosanu, G.: A proximal point algorithm converging strongly for general errors. Optim. Lett. 4, 635-641 (2010)

4. Boikanyo, O.A.: The viscosity approximation forward-backward splitting method for zeros of the sum of monotone operators. Abstr. Appl. Anal. 2016, 2371857 (2016). https://doi.org/10.1155/2016/2371857

5. Bruck, R.E.; Passty, G.B.: Almost convergence of the infinite product of resolvents in Banach spaces. Nonlinear Anal. 3, 279-282 (1979)

6. Burachik, R.S.; Scheimberg, S.: A proximal point method for the variational inequality problem in Banach spaces. SIAM J. Control Optim. 39, 1633-1649 (2000)

7. Chang, S.S.; Lee, H.J.; Chan, C.K.: A new method for solving equilibrium problem fixed point problem and variational inequality problem with application to optimization. Nonlinear Anal. Theory Methods Appl. 70(9), 3307-3319 (2009)

8. Cho, S.Y.; Qin, X.; Kang, S.M.: Iterative processes for common fixed points of two different families of mappings with applications. J. Glob. Optim. 57, 1429-1446 (2013)

9. Cioranescu, I.: Geometry of Banach Spaces, Duality Mappings and Nonlinear Problems, vol. 62. Mathematics and Its ApplicationsKluwer Academic Publishers, Dordrecht (1990)

10. Dadashi, V.: Shrinking projection algorithms for the split common null point problem. Bull. Aust. Math. Soc. 96, 299-306 (2017)

11. Dadashi, V.: On a hybrid proximal point algorithm in Banach spaces. Univ. Politeh. Buchar. Ser. A 80(3), 45-54 (2018)

12. Dadashi, V.; Khatibzadeh, H.: On the weak and strong convergence of the proximal point algorithm in reflexive Banach spaces. Optimization 66(9), 1487-1494 (2017)

13. Dadashi, V.; Postolache, M.: Hybrid proximal point algorithm and applications to equilibrium problems and convex programming. J. Optim. Theory Appl. 174(2), 518-529 (2017)

14. Goebel, K.; Kirk, W.A.: Topics in Metric Fixed Point Theory, vol. 28. Cambridge Studies in Advanced MathematicsCambridge University Press, Cambridge (1990)

15. Hadjisavvas, N.I.; Khatibzadeh, H.: Maximal monotonicity of bifunctions. Optimization 59(2), 147-160 (2010)

16. Kamimura, S.; Takahashi, W.: Weak and strong convergence of solutions to accretive operator inclusions and applications. Set Valued Anal. 8, 361-374 (2000)

17. Kang, S.; Cho, S.; Liu, Z.: Convergence of iterative sequences for generalized equilibrium problems involving inverse-strongly monotone mappings. J. Inequal. Appl. 2010(1), 827082 (2010)

18. Khatibzadeh, H.: Some remarks on the proximal point algorithm. J. Optim. Theory Appl. 153, 769-778 (2012)

19. Kinderlehrer, D.; Stampacchia, G.: An Introduction to Variational Inequalities and Their Applications. Academic Press, New York (1980)

20. Li, L.; Song, W.: Modified proximal point algorithm for maximal monotone operators in Banach spaces. J. Optim. Theory Appl. 138, 45-64 (2008)

21. Liu, L.S.: Ishikawa and Mann iterative process with errors for nonlinear strongly accretive mappings in Banach spaces. J. Math. Anal. Appl. 194(1), 114-125 (1995)

22. Lv, S.: Generalized systems of variational inclusions involving (A, $\eta$ )-monotone mappings. Adv. Fixed Point Theory 1(1), 15 (2011)

23. Martinet, B.: Régularisation d'inéquations variationnelles par approximations successives. Rev. Française Informat. Recherche Opérationnelle 3, 154-158 (1970)

24. Matsushita, S.; Xu, L.: On convergence of the proximal point algorithm in Banach spaces. Proc. Am. Math. Soc. 139, 4087-4095 (2011)

25. Moudafi, A.: On the regularization of the sum of two maximal monotone operators. Nonlinear Anal. Theory Methods Appl. 42(7), 1203-1208 (2000)

26. Moudafi, A.: On the convergence of the forward-backward algorithm for null-point problems. J. Nonlinear Var. Anal. 2, 263-268 (2018)

27. Moudafi, A.; Oliny, M.: Convergence of a splitting inertial proximal method for monotone operators. J. Comput. Appl. Math. 155(2), 447-454 (2003)

28. Moudafi, A.; Thera, M.: Finding a zero of the sum of two maximal monotone operators. J. Optim. Theory Appl. 94(2), 425-448 (1997)

29. Nadezhkina, N.; Takahashi, W.: Weak convergence theorem by an extragradient method for nonexpansive mappings and monotone mappings. J. Optim. Theory Appl. 128, 191-201 (2006)

30. Passty, G.B.: Ergodic convergence to a zero of the sum of monotone operators in Hilbert space. J. Math. Anal. Appl. 72(2), 383-390 (1979)

31. Qin, X.; Su, Y.: Approximation of a zero point of accretive operator in Banach spaces. J. Math. Anal. Appl. 329, 415-424 (2007)

32. Qin, X.; Cho, Y.J.; Kang, S.M.: Convergence theorems of common elements for equilibrium problems and fixed point problems in Banach spaces. J. Comput. Appl. Math. 225(1), 20-30 (2009)

33. Qin, X.; Cho, S.Y.; Wang, L.: A regularization method for treating zero points of the sum of two monotone operators. Fixed Point Theory Appl. 2014, 75 (2014). https://doi.org/10.1186/1687-1812-2014-75

34. Rockafellar, R.: On the maximal monotonicity of subdifferential mappings. Pac. J. Math. 33(1), 209-216 (1970)

35. Rockafellar, R.T.: Maximal monotone operators and proximal point algorithm. SIAM J. Control Optim. 14, 877-898 (1976)

36. Rouhani, B.D.; Khatibzadeh, H.: On the proximal point algorithm. J. Optim. Theory Appl. 137, 411-417 (2008) 
37. Schu, J.: Weak and strong convergence to fixed points of a asymptotically nonexpansive mappings. Bull. Aust. Math. Soc. 43, 153-159 (1991)

38. Suzuki, T.: Strong convergence theorems for infinite families of nonexpansive mappings in general Banach spaces. Fixed Point Theory Appl. 2005, 103-123 (2005)

39. Takahashi, W.: Approximating solutions of accretive operators by viscosity approximation methods in Banach spaces. In: Applied Functional Analysis, pp. 225-243, Yokohama Publishers, Yokohama (2007)

40. Takahashi, S.; Takahashi, W.; Toyoda, M.: Strong convergence theorems for maximal monotone operators with nonlinear mappings in Hilbert spaces. J. Optim. Theory Appl. 147(1), 2741 (2010)

41. Tian, C.; Wang, F.: The contraction-proximal point algorithm with square-summable errors. Fixed Point Theory Appl. 2013, 93 (2013). https://doi.org/10.1186/1687-1812-2013-93

42. Tseng, P.: A modified forward-backward splitting method for maximal monotone mappings. SIAM J. Control Optim. 38, 431-446 (2000)

43. Wang, F.; Cui, H.: On the contraction-proximal point algorithms with multi-parameters. J. Glob. Optim. 54(3), 485-491 (2012)

44. Wang, F.; Cui, H.: Convergence of the generalized contraction-proximal point algorithm in a Hilbert space. Optimization 64(4), 709-715 (2015)

45. Yao, Y.; Noor, M.A.: On convergence criteria of generalized proximal point algorithms. J. Comput. Appl. Math. 217(1), 46-55 (2008)

46. Yao, Y.; Shahzad, N.: Strong convergence of a proximal point algorithm with general errors. Optim. Lett. 6(4), 621-628 (2012)

47. Yuan, H.: A splitting algorithm in a uniformly convex and 2-uniformly smooth Banach space. J. Nonlinear Funct. Anal. 2018, 26 (2018). https://doi.org/10.23952/jnfa.2018.26

48. Zhang, S.; Lee, J.H.W.; Chan, C.K.: Algorithms of common solutions to quasi variational inclusion and fixed point problems. Appl. Math. Mech. Engl. Ed. 29(5), 571581 (2008)

Publisher's Note Springer Nature remains neutral with regard to jurisdictional claims in published maps and institutional affiliations. 